\title{
LMA Supreme for neonatal resuscitation: study protocol for a randomized controlled trial
}

\author{
Daniele Trevisanuto ${ }^{1,2^{*}}$, Francesco Cavallin ${ }^{3}$, Veronica Mardegan ${ }^{1}$, Nguyen Ngoc Loi ${ }^{4}$, Nguyen Viet Tien ${ }^{4}$, \\ Tran Dieu Linh ${ }^{4}$, Tran Dinh Chien ${ }^{5}$, Nicoletta Doglioni ${ }^{1}$, Lino Chiandetti ${ }^{1}$ and Luciano Moccia ${ }^{2,5}$
}

\begin{abstract}
Background: The most important action in the resuscitation of a newborn in the delivery room is to establish effective assisted ventilation. The face mask and endotracheal tube are the devices used to achieve this goal. Laryngeal mask airways that fit over the laryngeal inlet have been shown to be effective for ventilating newborns at birth and should be considered as an alternative to facemask ventilation or endotracheal intubation among newborns weighing $>2,000 \mathrm{~g}$ or delivered $\geq 34$ weeks' gestation. A recent systematic review and meta-analysis of supraglottic airways in neonatal resuscitation reported the results of four randomized controlled trials (RCTs) stating that fewer infants in the group using laryngeal mask airways required endotracheal intubation (1.5\%) compared to the group using face masks (12.0\%). However, there were methodological concerns over all the RCTs including the fact that the majority of the operators in the trials were anesthesiologists.

Our hypothesis is based on the assumption that ventilating newborns needing positive pressure ventilation with a laryngeal mask airway will be more effective than ventilating with a face mask in a setting where neonatal resuscitation is performed by midwives, nurses, and pediatricians. The primary aim of this study will be to assess the effectiveness of the laryngeal mask airway over the face mask in preventing the need for endotracheal intubation.

Methods/design: This will be an open, prospective, randomized, single center, clinical trial. In this study, 142 newborns weighing $>1,500 \mathrm{~g}$ or delivered $\geq 34$ weeks gestation needing positive pressure ventilation at birth will be randomized to be ventilated with a laryngeal mask airway (LMA Supreme ${ }^{\text {TM }}$, LMA Company, UK - intervention group) or with a face mask (control group). Primary outcome: Proportion of newborns needing endotracheal intubation. Secondary outcomes: Apgar score at 5 minutes, time to first breath, onset of the first cry, duration of resuscitation, death or moderate to severe hypoxic-ischemic encephalopathy within 7 days of life.
\end{abstract}

Trial registration: ClinicalTrials.gov identifier: NCT01963936 (October 11, 2013).

Keywords: Laryngeal mask, Resuscitation, Positive pressure ventilation, Newborn infant

\section{Background}

The ability to maintain a patent airway and provide effective positive pressure ventilation (PPV) is the main objective of neonatal resuscitation [1,2]. This is currently achieved with the use of a face mask (FM) or an endotracheal tube (ETT). Both these devices have major limitations from a strictly anatomical point of view and require adequate operator skills. In certain situations, both FM

\footnotetext{
* Correspondence: trevo@pediatria.unipd.it

'Department of Women and Children Health, University of Padua, Via Giustiniani, 3, Azienda Ospedaliera di Padova, Padova 35128, Italy

${ }^{2}$ Amici della Neonatologia Trentina, Trento, Italy

Full list of author information is available at the end of the article
}

ventilation and ETT intubation may prove difficult to establish an upper airway [3,4].

In 1981, Archie Brain designed the laryngeal mask airway (LMA) with the aim of producing an airway device that would be more practical than the FM and less invasive than the ETT [5].

In adults, the LMA is routinely used during anesthesiology procedures.

The potential advantages of the LMA over the FM include an easier insertion technique, less manipulation of the patient's head, neck, and jaw, a better airtight seal after positioning, and more effective PPV [6]. Ease of positioning and reduced invasiveness are the reported 
advantages of the LMA when compared to the tracheal tube [6].

A recent study including 11,910 anesthesia pediatric cases showed that only 102 cases (0.86\%) experienced LMA failure. Common presenting features of LMA failures included leak (25\%), obstruction (48\%), and patient intolerance such as intractable coughing/bucking (11\%) [7].

In the setting of the neonatal resuscitation, previous observational studies showed that, when used by teams with expertise (that is, anesthesiologists), the LMA provided effective PPV in most of the treated patients (range 95 to 99\%) [8-10]. A 2005 Cochrane review concluded that there was no evidence to evaluate the safety or efficacy for the use of LMA versus FM ventilation in the resuscitation of newborn infants [11]. It suggested that a well-designed randomized controlled trial (RCT) comparing these two airway adjuncts was warranted [11]. In 2013, a systematic review and meta-analysis of supraglottic airways in neonatal resuscitation reported the results of four RCTs, stating that fewer infants in the LMA group required endotracheal intubation (1.5\%) compared to the FM group (12.0\%) [12]. There were however, methodological concerns over all the RCTs including the fact that the majority of the operators in the trials were anesthesiologists (who are less likely in most clinical settings to be present at neonatal resuscitation).

The LMA is used more often by anesthesiologists rather than pediatricians, nurses, and midwives. It is thus essential to demonstrate its effectiveness by those who will be more commonly involved in neonatal resuscitation in most clinical settings. Furthermore, it is important to thoroughly record any side effects, as in a case series of a comparison of LMA over FM in infants in the operating theatre, significantly more side effects were reported using the LMA over the FM $[13,14]$.

Although previous studies showed that the LMA was effective also in preterm infants weighing less than 2,000 $\mathrm{g}$ $[9,10]$, the last version of the International Guidelines for Neonatal Resuscitation state that "a LMA should be considered during resuscitation if FM ventilation is unsuccessful and tracheal intubation is unsuccessful or not feasible. The LMA may be considered as an alternative to a FM for PPV among newborns weighing $>2,000 \mathrm{~g}$ or delivered $\geq 34$ weeks' gestation" [1,2].

Despite this recommendation, it has not yet been shown in a well-conducted RCT whether or not LMA is more effective than FM in resuscitation of newborn infants.

\section{Methods/design}

Aim

The primary aim of this study will be to assess the effectiveness of LMA over FM ventilation in preventing the need for endotracheal intubation at birth.

\section{Study design}

This is a single center, prospective, unblinded, randomized clinical trial of LMA ventilation versus FM ventilation in infants weighing $>1,500 \mathrm{~g}$ or delivered $\geq 34$ weeks' gestation.

\section{Inclusion criteria}

Inborn infants satisfying the following inclusion criteria will be eligible to participate in the study:

1. gestational age $\geq 34$ weeks (and)

2. expected birth weight $>1,500 \mathrm{~g}[9,10]$ (and)

3. need for PPV at birth; the need for PPV will be determined by the presence of apnoea or gasping, or heart rate $<100$ beats per minute (bpm) after initial resuscitation measures (providing warmth, positioning, clearing the airway, drying and stimulation) over the first 30 seconds [1,2] (and)

4. parental consent; a written informed consent will be obtained by a member of the neonatal team involved in the study from a parent or guardian before delivery.

\section{Exclusion criteria}

1. Lethal anomalies.

2. Hydrops.

3. Major malformations of the respiratory system.

4. Congenital heart disease.

5. Stillbirths; a stillbirth will be diagnosed when a heart rate is never established.

\section{Primary outcome measure}

The primary outcome of this study will be the proportion of newborns needing endotracheal intubation.

\section{Secondary outcome measures}

1. Apgar score at 5 minutes.

2. Time to first breath, defined as the first respiratory effort.

3. Time to first cry, defined as the first audible cry spontaneously emitted by the infant.

4. Death or moderate to severe hypoxic-ischemic encephalopathy (HIE) within 7 days of life, according to a modification of Sarnat and Sarnat $[15,16]$. According to this classification, HIE grade I (mild) includes irritability, hyperalertness, mild hypotonia, and poor sucking; grade II (moderate) includes lethargy, seizures, marked abnormalities of tone, and requirement of tube feeding; grade III (severe) includes coma, prolonged seizures, severe hypotonia, and failure to maintain spontaneous respiration.

5. Complications secondary to the procedure.

6. Admission to NICU/normal nursery. 


\section{Other collected data}

The following data will be collected during resuscitation: (1) Apgar score at 1 min after birth; (2) LMA insertion time, the rate of successful insertion at the first attempt, and the number of attempts required to insert the LMA successfully; (3) adverse effects during resuscitation.

\section{Generalizability}

The findings of this study will be important for other units/settings in high as well low resource countries where neonatal resuscitation is more often performed by pediatricians, midwives, or nurses. Based on the results of the present study, we could speculate whether a short-term educational program on the LMA use will be effective in the clinical practice; furthermore, we will be able to understand whether personnel involved in neonatal resuscitation should be trained to start resuscitation with an FM or with an LMA. Finally, potential complications and side effects due to the LMA will be strictly monitored and collected.

\section{Sample size}

It is estimated that $5 \%$ of newborns receive resuscitation with PPV [1,2]. Therefore, in C hospital, Hanoi, Vietnam with more than 20,000 deliveries per year, approximately 1,000 patients will require resuscitation. Of these, about $90 \%$ (900 neonates) are newborns weighing $>1,500$ g or delivered $\geq 34$ weeks gestation.

The sample size was based on a previous study in which the success rate of LMA and FM were $99 \%$ and $84 \%$, respectively [17]. To obtain a $90 \%$ power at a 0.05 level of significance (one-sided), at least 58 subjects per group need to be enrolled. The number of patients was increased by $20 \%$ for each group considering the possibility of dropouts, leading to a final sample of 142 subjects.

\section{Recruitment}

Written and oral information will, whenever possible, be offered to parents prior to the birth of their child if the infant is likely to be eligible. Informed written consent will be signed by a parent or guardian. A senior investigator will be available at all times to discuss concerns raised by parents or clinicians during the course of the trial.

\section{Randomization}

Eligible infants will be assigned to the LMA or the FM group in a 1:1 ratio according to a computer-generated, randomized sequence. The randomized allocation will be concealed in double-enclosed, opaque, sealed, and sequentially numbered envelopes prepared at the University Hospital of Padua.
In the delivery room or operating room, the next sequential randomization envelope will be opened only when the infant will be considered to be eligible by the attending operator. The assigned procedure (PPV with LMA or FM) will then be performed. Multiple births will be separately randomized.

\section{Blinding}

Due to the characteristics of the intervention, neither caregivers nor outcome assessors will be masked to treatment allocation. To minimize bias, strict criteria and definitions will be maintained during the trial.

\section{Guidelines for management}

Before starting the study, all those involved in the neonatal resuscitation participated in a one-day theoretical and practical (manikin) course based on the Neonatal Resuscitation Program (NRP). During the course, one section was dedicated to the preparation and insertion of the size $1 \mathrm{Su}$ preme LMA $^{\mathrm{Tm}}$ [18]. In November 2011, three courses were held by three certificated NRP teachers in collaboration with an expert in the LMA use (DT). A total of 44 participants (15 physicians and 29 nurses) were trained.

After the course, a period of 3 months was left to routinely introduce LMA use in the delivery rooms. Five successful LMA insertions in the manikin and three LMA insertions in the clinical setting were required to all participants before starting the study.

The American Heart Association and American Academy of Pediatrics Guidelines for Neonatal Resuscitation will be followed in this study [1,2]. After initial steps (warming, clearing airway, drying, stimulation), PPV with FM or LMA and bag will be initiated in the case of apnea and/or gasping and/or heart rate $<100 \mathrm{bpm}$. The neonate's trachea will be intubated if the heart rate does not rise or remains less than $60 \mathrm{bpm}$ after 30 seconds of PPV with the LMA or FM. A maximum of three attempts for obtaining effective PPV with an LMA or an FM will be allowed.

Manual ventilation will be initiated in room air at a frequency of 40 to 60 breaths per minute [1,2]. The $\mathrm{FiO}_{2}$ will be increased to 1,0 (flow rate 6 to $8 \mathrm{~L} / \mathrm{min}$ ) in case of persistent cyanosis and/or heart rate $<100 \mathrm{bpm}$ after 90 seconds from the beginning of PPV. At least two trained people involved in the study will take part in the resuscitation of all enrolled patients.

Resuscitation will start immediately after delivery of the infant, when a stop watch will be switched on by one of the members of the resuscitation team.

The duration of resuscitation will be defined as the time period from starting resuscitation to the establishment of a spontaneous and sustained respiratory pattern of efficacious respiratory movements, which allowed the 
neonate to maintain adequate clinical parameters (heart and respiratory rate).

In this study, the last model of size 1 LMA Supreme (LMA Supreme $^{\mathrm{Tm}}$, LMA Company, UK) will be used [18]. Previous studies conducted in adult patients showed the efficacy and the safety of the LMA Supreme [19,20]. The LMA Supreme is superior to the LMA Classic ${ }^{\mathrm{TM}}$ with regard to insertion time and oropharyngeal seal pressure [21]; a further advantage consists in the gastric access.

A previous neonatal manikin study confirmed these findings, including a higher level of satisfaction expressed by users [22].

\section{Data collection}

Data will be recorded from clinical records and from a data sheet designed for this study, where all the data obtained during resuscitation procedures will be collected by an observer not involved in the resuscitation maneuvers. Registered clinical information will be: eligibility, antenatal history, randomization, and all data above listed in the 'Primary outcome measure', 'Secondary outcome measures' and 'Other collected data' sections. Further information will be collected on expected serious adverse events (SAEs).

\section{Statistical analysis}

Categorical data will be expressed as number and percentage and compared using Fisher's test. Continuous data will be expressed as mean and standard deviation or median and interquartile range. The normality assumption of continuous variables will be evaluated using Shapiro-Wilk test. Continuous data will be compared using Student's $t$-test or the Mann-Whitney non-parametric test. Correlation between continuous data will be evaluated using the Pearson correlation coefficient or the Spearman correlation coefficient. A $P$-value less than 0.05 will be considered significant. Statistical analysis will be performed using the R 2.12 language [23].

\section{Duration of study}

In this study, 142 infants will be recruited. The trial will terminate when the last recruited infant is discharged from hospital, or dies.

\section{Ethics committee approval}

The C hospital, Hanoi, Vietnam Ethis Committees for $\mathrm{Hu}-$ man Investigation approved the study (SO:901/QD-PSTW; Ha Noi, 9 August 2012).

\section{Compliance to protocol}

Compliance will be defined as full adherence to protocol. Compliance with the protocol will be ensured by two members of the project (TDC, NTTH) responsible for local data collection. They will weekly monitor the adherence to the study protocol and will input the patients' data in an Excel data sheet.

\section{Safety}

Safety measures will include incidence, severity, and causality of reported SAEs, represented by changes in occurrence of the expected common neonatal complications and the development of unexpected SAEs. All SAEs will be followed until complete resolution or until the clinician responsible for the care of the recruited patient considers the event to be chronic or the infant to be stable. If there is a reasonable suspected causal relationship with the intervention, SAEs will be reported to the Ethics Committee to guarantee the safety of the participants.

\section{Discussion}

There are unique features of this trial compared to prior studies on the use of the LMA during neonatal resuscitation. To our knowledge, three randomized controlled trials including 140 patients have been previously published [12]. Due to the limited number of enrolled patients, a final conclusion cannot be drawn. A further trial with a different study design (quasi- randomized controlled trial) showed that the LMA significantly reduced the need of intubation in the delivery room in comparison with FM ventilation [17]. All these studies were performed with a classic LMA. In this trial, we used, for the first time, a more advanced model of LMA, the LMA Supreme.

\section{Trial status}

The trial is currently recruiting study subjects.

\section{Abbreviations}

BPM: beats per minute; ETT: endotracheal tube; FM: face mask; HIE:

hypoxic-ischemic encephalopathy; LMA: laryngeal mask airway; NRP: Neonatal Resuscitation Program; PPV: positive pressure ventilation; SAEs: serious adverse events; SLMA: supreme laryngeal mask airway.

\section{Competing interests}

The authors declare that they have no competing interests.

\section{Authors' contributions}

DT, FC, VM, NNL, NVT, TDL, TDC, ND, LC, and LM have made substantial contributions to the conception and design of the study protocol, and have given final approval of the version to be published. All authors read and approved the final manuscript.

\section{Acknowledgments}

We thank Laryngeal Mask Airway Co. Ltd, Jersey, UK, which will provide the SLMA that will be used in this study. The LMA Company was not involved in the study conception and design and will not be involved in the analysis and interpretation of the study results.

We did not receive external funding for this study.

\section{Author details}

${ }^{1}$ Department of Women and Children Health, University of Padua, Via Giustiniani, 3, Azienda Ospedaliera di Padova, Padova 35128, Italy. ${ }^{2}$ Amici della Neonatologia Trentina, Trento, Italy. ${ }^{3}$ Independent statistician, Padova, Italy. ${ }^{4}$ Department of Neonatal Intensive Care, National Hospital of Obstetrics 
and Gynecology, Ha Noi, Vietnam. ${ }^{5}$ Breath of Life Program - East Meets West Foundation, Oakland, CA, USA.

Received: 8 November 2013 Accepted: 27 June 2014

Published: 15 July 2014

\section{References}

1. Perlman JM, Wyllie J, Kattwinkel J, Atkins DL, Chameides L, Goldsmith JP, Guinsburg R, Hazinski MF, Morley C, Richmond S, Simon WM, Singhal N, Szyld E, Tamura M, Velaphi S, Neonatal Resuscitation Chapter Collaborators: International consensus on cardiopulmonary resuscitation and emergency cardiovascular care science with treatment recommendations. Pediatrics 2010, 2010(126):e1319-e1344.

2. Kattwinkel J, Perlman JM, Aziz K, Colby C, Fairchild K, Gallagher J, Hazinski MF, Halamek LP, Kumar P, Little G, McGowan JE, Nightengale B, Ramirez MM, Ringer S, Simon WM, Weiner GM, Wyckoff M, Zaichkin J, American Heart Association: Neonatal resuscitation, American Heart Association guidelines for cardiopulmonary resuscitation and emergency cardiovascular care. Pediatrics 2010, 2010(126):e1400-e1413.

3. O'Donnell CP, Kamlin CO, Davis PG, Morley CJ: Endotracheal intubation attempts during neonatal resuscitation: success rates, duration, and adverse effects. Pediatrics 2006, 117:e16-e21.

4. Doglioni N, Cavallin F, Zanardo V, Trevisanuto D: Intubation training in neonatal patients: a review of one trainee's first 150 procedures. J Matern Fetal Neonatal Med 2012, 25:1302-1304.

5. Al B: The laryngeal mask-a new concept in airway management. $\mathrm{Br}$ J Anaesth 1983, 55:801-805.

6. Trevisanuto D, Micaglio M, Ferrarese $P$, Zanardo V: The laryngeal mask airway: potential application in neonates. Arch Dis Child Fetal Neonatal Ed 2004, 89:F485-F489.

7. Paterson SJ, Byrbe PJ, Molesky MG, Seal RF, Finucane BT: Neonatal resuscitation using the laryngeal mask airway. Anesthesiology 1994 80:1248-1253.

8. Gandini D, Brimacombe JR: Neonatal resuscitation with the laryngeal mask airway in normal and low birth weight infants. Anesth Analg 1999, 89:642-643.

9. Trevisanuto D, Micaglio M, Pitton M, Magarotto M, Piva D, Zanardo V: Laryngeal mask airway: is the management of neonates requiring positive pressure ventilation at birth changing? Resuscitation 2004 62:151-157.

10. Zanardo V, Weiner G, Micaglio M, Doglioni N, Buzzacchero R, Trevisanuto D: Delivery room resuscitation of near-term infants: role of the laryngeal mask airway. Resuscitation 2010, 81:327-330.

11. Grein AJ, Weiner GM: Laryngeal mask airway versus bag-mask ventilation or endotracheal intubation for neonatal resuscitation. Cochrane Database Syst Rev 2005, 2:CD003314

12. Schmölzer GM, Agarwal M, Kamlin CO, Davis PG: Supraglottic airway devices during neonatal resuscitation: an historical perspective, systematic review and meta-analysis of available clinical trials. Resuscitation 2013, 84:722-730.

13. Harnett M, Kinirons B, Heffernan A, Motherway C, Casey W: Airway complications in infants: comparison of laryngeal mask airway and the facemask-oral airway. Can J Anaesth 2000, 47:315-318.

14. Mathis MR, Haydar B, Taylor EL, Morris M, Malviya SV, Christensen RE, Ramachandran SK, Kheterpal S: Failure of the Laryngeal Mask Airway Unique $^{\mathrm{TM}}$ and Classic $^{\mathrm{TM}}$ in the pediatric surgical patient: a study of clinical predictors and outcomes. Anesthesiology 2013, 119:1284-1295.

15. Levene MI, Kornberg J, Williams THC: The incidence of and severity of postasphyxial encephalopathy in full-term infants. Early Hum Dev 1985, 11:21-28.

16. Sarnat HB, Sarnat MS: Neonatal encephalopathy following fetal distress. A clinical and electroencephalographic study. Arch Neurol 1976, 33:696-705

17. Zhu XY, Lin BC, Zhang QS, Ye HM, Yu RY: A prospective evaluation of the efficacy of the laryngeal mask airway during neonatal resuscitation. Resuscitation 2011, 82:1405-1409.

18. The LMA-Supreme ${ }^{\mathrm{TM}}$ : Instructions for Use. Jersey, UK: The Laryngeal Mask Company Limited, Teleflex Medical; 2013. http://www.Imaco.com/ catalogue_item.php?catID=5799\&prodID=56350 (last access July, 15, 2014).

19. van Zundert A, Brimacombe J: The LMA Supreme-a pilot study. Anaesthesia 2008, 63:209-210.
20. Cook TM, Gatward JJ, Handel J, Hardy R, Thompson C, Srivastava R, Clarke PA: Evaluation of the LMA Supreme in 100 non-paralysed patients. Anaesthesia 2009, 64:555-562.

21. Wong DT, Yang JJ, Jagannathan N: Brief review: the LMA Supreme ${ }^{\mathrm{TM}}$ supraglottic airway. Can J Anaesth 2012, 59:483-493.

22. Trevisanuto D, Parotto M, Doglioni N, Ori C, Zanardo V, Micaglio M: The Supreme Laryngeal Mask Airway ${ }^{\mathrm{TM}}$ (LMA): a new neonatal supraglottic device: comparison with Classic and ProSeal LMA in a manikin. Resuscitation 2012, 83:97-100.

23. R Development Core Team: A language and environment for statistical computing. Vienna, Austria: R Foundation for Statistical Computing; 2010 ISBN ISBN 3-900051-07-0.

\section{doi:10.1186/1745-6215-15-285}

Cite this article as: Trevisanuto et al: LMA Supreme for neonatal resuscitation: study protocol for a randomized controlled trial. Trials 2014 15:285

\section{Submit your next manuscript to BioMed Central and take full advantage of:}

- Convenient online submission

- Thorough peer review

- No space constraints or color figure charges

- Immediate publication on acceptance

- Inclusion in PubMed, CAS, Scopus and Google Scholar

- Research which is freely available for redistribution 